\title{
Model-based identification of PEEP titrations during different volemic levels
}

\author{
C. Starfinger ${ }^{1}$, J.G. Chase $^{1}$, C.E. Hann ${ }^{1}$, G.M.Shaw ${ }^{2}$, P. Lambert ${ }^{3}$, \\ B.W. Smith ${ }^{4}$, E. Sloth ${ }^{3}$, A. Larsson ${ }^{3}$, S. Andreassen ${ }^{4}$, S. Rees ${ }^{4}$ \\ 1 Centre for Bioengineering, University of Canterbury, Christchurch, New Zealand \\ ${ }^{2}$ Department of Intensive Care Medicine, Christchurch Hospital, Christchurch, New Zealand \\ 3 Department of Anesthesia, Emergency and Intensive Care Medicine, North Jutland, \\ Ålborg Hospital - Århus University Hospitals, Denmark \\ 4 Center for Model-based Medical Decision Support, Ålborg University, Denmark
}

February 25, 2008

\section{Abstract}

A cardiovascular system (CVS) model has previously been validated in simulated cardiac and circulatory disease states. It has also been shown to accurately capture all main hemodynamic trends in a porcine model of pulmonary embolism. In this research, a slightly extended CVS model and parameter identification process are presented and validated in a porcine experiment of positive end-expiratory pressure (PEEP) titrations at different volemic levels. The model is extended to more physiologically represent the separation of venous and arterial circulation. Errors for the identified model are within $5 \%$ 
when re-simulated and compared to clinical data. All identified parameter trends match clinically expected changes. This work represents another clinical validation of the underlying fundamental CVS model, and the methods and approach of using them for cardiovascular diagnosis in critical care.

Keywords: cardiovascular system, cardiac model, parameter identification, integral method, PEEP, hypovolemia

\section{Introduction}

Cardiac disease state is highly patient specific and difficult to accurately diagnose due to the limited measurements typically available. Therefore, successful diagnosis and treatment often rely on the experience and intuition

of clinical staff. A physiologically validated computer model could offer several advantages in diagnosis and therapy guidance, by aggregating diverse and less transparent patient data into an overall patient-specific, clinically relevant assessment of a patient's circulatory status.

In particular, a CVS model can be formulated in terms of equations whose parameters are physiologically relevant. When patient-specific parameters can be identified from measured clinical data so that the model matches the clinical measurements, a patient-specific model of their fundamental dynamic 
(Pressure-Volume, PV) behavior is created. These physiologically relevant parameters can then be used to assist clinical staff with diagnosis.

For example, the contractility index of the left ventricle can be elevated or depressed, thus giving insight into the performance of the ventricle. A similar example would be the parameter representing the pulmonary vascular resistance providing information about the pressures in the pulmonary arteries, to ascertain if an embolus is developing. More complex examples could include discerning the onset of septic or other forms of shock [1].

Therefore, by observing how patient-specific parameters change over time (new identifications can be performed for any few heartbeats of data) valuable information about the patient's hemodynamic condition can be tracked, diagnosed and monitored to help provide effective treatment. This use of the model has already been validated on clinical data from animal trials (pigs) in induced pulmonary embolism [2]. This research presents a further validation of the model and methods by identifying the different hemodynamic conditions that result from changing PEEP and volemic levels, which are common therapies in critical care that can have significant impact on circulatory function.

Spontaneous breathing and ventilation can have extensive effects on car- 
diovascular function. These effects are usually well tolerated in healthy subjects but may cause detrimental hemodynamic effects in critically ill patients, where pulmonary and or cardiovascular diseases and dysfunctions are present. It is thus important to understand the cardiopulmonary interactions and account for them when modelling the cardiovascular system.

Positive end-expiratory pressure (PEEP) is an operator-controlled variable that can be set during mechanical ventilation. The correct value of PEEP can be beneficial or detrimental for the patient dependent on how it is used. More specifically, in patients with lung injury, high PEEP levels may be necessary to maintain or restore oxygenation and for each individual patient the right balance between too much and too little PEEP has to be found. Too little PEEP may result in airway or alveolar collapse, whereas too much PEEP can cause alveolar overdistention and hemodynamic problems such as a reduction in cardiac output $(\mathrm{CO})$.

In this research, the CVS model is extended to more realistically capture different hemodynamic conditions resulting from the application of different PEEP values and levels of normo-, hypo- and hypervolemia. Compared to prior work, the minimal data required for the parameter identification process has been further reduced. The whole approach is thus more applicable for a 
clinical environment despite a modest model extension. In particular, instead of using the left and right ventricular systolic and diastolic volumes, only the global end-diastolic volume (GEDV) and an estimated total blood volume (TBV) are now needed to identify the different PEEP and volemic levels correctly. The identification process has also been modified from prior work to allow for a more robust identification.

\section{Methodology}

\subsection{CVS model}

The CVS model is a simple, yet clinically validated model for the heart and circulation [3]. This model comprises a series of connected pressure-volume chambers with greater detail for the active ventricular portions of the heart and their interaction. More specifically, the left/right ventricle, aorta, pulmonary artery/vein, vena cava are captured as explicit physiological regions with independent fundamental circulatory (PV) dynamics. A more detailed description of this foundation CVS model can be found in [2-4].

In this research, the original CVS model has been extended to include one extra compartment for the lung capillaries. A second has been added for the body capillaries, thus separating the venous and arterial systems and 
resistances. Figure 1 shows these changes with the two new compartments $P, V_{\text {sys }}$ and $P, V_{\text {cap }}$ which represent the systemic and pulmonary capillaries, respectively. However, for reasons of simplicity and avoiding unnecessary complexity, only 1 additional compartment was added for the pulmonary and systemic circulation, respectively.

This physiological addition is of great interest for heart-lung interactions during positive pressure ventilation (PPV), particularly when studying the effect of different PEEP levels. When PEEP is raised, increases in intrathoracic pressure $\left(P_{t h}\right)$ cause right ventricular preload to decrease by increasing the resistance to venous return $\left(R_{v r}\right)$, and left ventricular afterload to decrease $[5,6]$. This afterload reduction is achieved by a decrease in systemic

resistance $\left(R_{s y s}\right)$. One can see, that two resistances on either side, the arterial and venous side, are necessary to correctly simulate these complex behaviors observed clinically during mechanical or spontaneous breathing, thus physiologically justifying this model extension.

\subsection{Integral-Based Parameter Identification}

The parameter identification method used in this research has previously been shown to rapidly and accurately identify almost the entire parameter 
set in the presence of significant measurement noise $[2,7]$. This identification method is modified in this research to allow a more robust parameter identification with similar or lesser data requirements.

\subsubsection{Adjustments to arterial elastances $\left(E_{a o}, E_{p a}\right)$}

The main modifications to previous work include keeping the arterial elas-

tances $\left(E_{a o}, E_{p a}\right)$ fixed, thus allowing other parameters to be more easily and accurately calculated. Arterial compliance is defined as the change in volume following a change in pressure. Arterial elastance is given as the reciprocal of the compliance and thus by a pressure change $(\triangle P)$ following a volume change $(\triangle V)$. Many researchers have concluded, that arterial elastance can consequently be calculated by substituting the pulse pressure (PP) for $\triangle P$ and stroke volume (SV) for $\triangle V$ as given by following equations [8, 9]:

$$
\begin{aligned}
& E_{a o}=\frac{P P_{a o}}{S V} \\
& E_{p a}=\frac{P P_{p a}}{S V}
\end{aligned}
$$

Hence, $E_{a o}$ and $E_{p a}$ are no longer identified, but are directly obtained from the measured arterial and pulmonary artery pulse pressures $\left(P P_{a o}, P P_{p a}\right)$ and stroke volumes. If $P_{p a}$ is not measured, $E_{a o}$ is still known as it can be 
assumed that $P_{a o}$ and SV are almost always measured in acute critical care patients. Note that, in this research, Equations 1 and 2 are adjusted by a multiplying factor of 1.25 to account for dynamic effects in static measures, as it was found from observing the relationship between $\mathrm{SV}$ and $V_{a o} / V_{p a}$, that adding this factor improves the estimate for $E_{a o}$ and $E_{p a}$. Otherwise both elastances would be slightly underestimated. Consequently, the two parameters, systemic vascular resistance and elastance $\left(R_{\text {sys }}, E_{\text {sys }}\right)$ can thus be determined directly using:

$$
\begin{aligned}
R_{s y s} & =\frac{E_{a o} \cdot\left(\int P_{s y s}-\int P_{a o}\right)}{\left(P_{a o}-P_{a o 0}-\int Q_{a v} \cdot E_{a o}\right)} \\
E_{\text {sys }} & =\frac{\int P_{a o}-R_{s y s} \cdot\left(\int Q_{a v}-1 / E_{a o} \cdot\left(P_{a o}-P_{a o 0}\right)\right)}{\int V_{s y s}}
\end{aligned}
$$

where the integral is taken from measured data (numerically). Similarly, pulmonary vascular resistance and elastance $\left(R_{\text {pulin }}\right.$ and $\left.E_{c a p}\right)$ can also be found:

$$
\begin{aligned}
R_{p u l i n} & =\frac{E_{p a} \cdot\left(\int P_{c a p}-\int P_{p a}\right)}{\left(P_{p a}-P_{p a 0}-\int Q_{p v} \cdot E_{p a}\right)} \\
E_{c a p} & =\frac{\int P_{p a}-R_{p u l i n} \cdot\left(\int Q_{p v}-1 / E_{p a} \cdot\left(P_{p a}-P_{p a 0}\right)\right)}{\int V_{c a p}}
\end{aligned}
$$

Note that for reasons of clarity, the differential $\mathrm{d} t$, and upper and lower limits 
of integration are omitted. If not stated otherwise, the integration is done over at least one heart beat. In cases where matrices are constructed separately for ejection and filling periods, the integrals are only calculated during these periods. More detailed information about this part of the identification process can be found in $[2,7]$.

\subsubsection{Scaling process}

Where the waveforms are not known and only discrete measurements are available the integral method of [7] cannot be directly applied. However, waveforms can be artificially generated by scaling a set of previously calculated model outputs to best fit the discrete maximum and minimum measured data values over one or more heart beats for the pressures and volumes. The assumption is that these validated model waveforms are reasonably conformable with the actual clinical case.

These scaled signals are then re-identified and a new CVS forward simulation is performed with the previously identified parameters producing a much closer match to the clinical data than the first initial, assumed parameter set. The simulated output is then compared to the clinical data. Subsequently, the output signals are re-scaled and new parameters are identified that are then again used to run another simulation. This iterative process is stopped 
when the relative error between model output and clinical data reaches a set tolerance.

\subsubsection{Substitution of flow integrals during the scaling process}

Another modification to the methods of $[2,7]$ has been made to better calculate the parameters that are determined by the flows in and out of the ventricles. Previously, these flows have been used in the identification process. This approach introduced significant error during the scaling process, where only the pressure and volume signals, but not the unknown associated flows, are scaled. To address this issue, the flow integrals $\int Q_{m t}, \int Q_{a v}, \int Q_{t c}$ and $\int Q_{p v}$ are now substituted by their corresponding volumes, as given in Equations 7 - 10 .

$$
\begin{gathered}
V_{l v}(e f)-V_{l v}(e b)=-\int_{e b}^{e f} Q_{a v} \mathrm{~d} t \\
V_{l v}(f f)-V_{l v}(f b)=\int_{f b}^{f f} Q_{m t} \mathrm{~d} t \\
V_{r v}(e f 2)-V_{r v}(e b 2)=-\int_{e b 2}^{e f 2} Q_{p v} \mathrm{~d} t \\
V_{r v}(f f 2)-V_{r v}(f b 2)=\int_{f b 2}^{f f 2} Q_{t c} \mathrm{~d} t
\end{gathered}
$$

with $e b, e b 2=$ ejection begin for $\mathrm{LV}, \mathrm{RV}$; ef, ef $2=$ ejection finish for $\mathrm{LV}, \mathrm{RV}$; $f b, f b 2=$ filling begin for $\mathrm{LV}, \mathrm{RV}$ and $f f, f f 2=$ filling finish for $\mathrm{LV}, \mathrm{RV}$. This 
approach has the advantage that the volumes in the ventricles are either measured or estimated signals, whereas the flows are usually not measured or estimated. Hence, significantly less error is introduced during the scaling part of the parameter identification process using these discrete measurements. Figure 2 shows in the upper panel the flow integral and corresponding volume signal for $Q_{a v}$. The lower panel shows the difference between both signals which is negligible.

\subsection{Volume Calculations}

The parameter identification process needs the two ventricle volumes as input signals to accurately determine some of the parameters. More specifically, the end-diastolic and end-systolic volumes (EDV, ESV) are needed. However, these volume measurements are usually not available in a clinical environment and thus need to be estimated from the clinically available data.

Currently, the LVEDV and RVEDV are estimated based on an estimated total blood volume (TBV) and the measured global end-diastolic volume (GEDV). As the stroke volume (SV) is also a measured variable, ESV can be

calculated by subtracting SV from EDV. The total blood volume is estimated as $85 \mathrm{ml} / \mathrm{kg}$, with $25 \mathrm{ml} / \mathrm{kg}$ being stressed volume and $60 \mathrm{ml} / \mathrm{kg}$ unstressed 
volume based on clinical results [10].

GEDV is the end-diastolic volume of the left and right ventricle and the two atria. Comparing the given GEDV measurements for the pigs in this study with typical porcine ventricular volume measurements from other studies $[2,11-13]$, it can be seen that GEDV seems to be slightly overestimated here. Hence, the GEDV used in this research is corrected by a factor $f=2 / 3$ to produce commonly seen LVEDV and RVEDV values for healthy pigs based on their weight. Furthermore, from these studies it can also be assumed that the right ventricle usually contains more blood than the left ventricle, especially in the case where a high PEEP value is present [14]. This estimation is further validated by several studies using angiography to measure the ventricle volumes and it has been demonstrated that the normal left-ventricular end-diastolic index (LVEDVI) in man is 70 to $80 \mathrm{~mL} / \mathrm{m}^{2}$, whereas the mean right-ventricular end-diastolic volume index (RVEDVI) is 80 to $85 \mathrm{~mL} / \mathrm{m}^{2}$ [15-17]. Additionally, it can be estimated that within the heart, blood is distributed with $60 \%$ in the ventricle and $40 \%$ in the atrium [18]. Hence the LVEDV and RVEDV are estimated by following equations: 


$$
\begin{aligned}
& L V E D V=2 / 3 \cdot G E D V \cdot 0.6 \cdot 0.4 \\
& R V E D V=2 / 3 \cdot G E D V \cdot 0.6 \cdot 0.6
\end{aligned}
$$

The remaining model volumes can be estimated based on known blood distributions. Importantly, the volume in the pulmonary capillary and vein compartments $\left(V_{c a p}, V_{p v}\right)$ make up the entire pulmonary blood volume (PBV), which can be estimated as $G E D V / 4$ [19]. The volumes in the aorta and pulmonary artery are directly obtained from the measured pressures $P_{a o}$ and $P_{p a}$ and the calculated elastances $E_{a o}$ and $E_{p a}$. These two volumes are thus defined by the simple definition:

$$
V=\frac{P}{E}
$$

Note that for simplicity, the unstressed volumes are omitted, except for the systemic and pulmonary volume compartments $\left(V_{\text {sys }}, V_{\text {cap }}\right)$, which both are modelled using stressed and unstressed volumes. Further, as a result of these estimations, specific detailed questions cannot be answered, as information is lost on how one ventricle expands relative to the other. For example, the RVEDV/LVEDV expansion index is no longer valid due to these assump- 
tions. However, this research focuses on identifying the change in SV, which is directly given as a measured signal, rather than identifying specific ventricular volumes or related dysfunction. Thus, this identification method is specific to capturing these circulatory effects due to changes in PEEP, which is a relatively common occurrence in the ventilated critical care patient.

\subsection{Summary of the identification process}

Figure 3 gives an overview of the identification process, which is based on previous work $[2,7]$. Modifications to the overall process are described above. The following steps are performed:

1. Obtain clinical measurements and signals as shown in Table 1

2. Use volume calculations to estimate the initial volume conditions for the CVS model

3. Use initial set of parameters to obtain first simulation output [2]

4. Scale simulation output signals $\left(P_{p a}, P_{a o}, V_{l v}, V_{r v}\right)$ to match the clinical, porcine data $[2]$

5. Identify the animal-specific parameters for the scaled signals using the integral-based methods based on [7] 
6. Re-scale the simulation output signals to better match the porcine data

7. Repeat steps 4 to 6

8. Stop the iterative process when a set error tolerance is reached

\subsection{PEEP experiment study protocol}

\subsubsection{Instrumentation and Monitoring}

The experiment was approved by the Danish National animal ethics committee and data of seven $20-22 \mathrm{~kg}$ pigs was analyzed for this research. A detailed description of the anesthesia, ventilation, instrumentation and monitoring procedure has been published in [20]. Thus, only a brief discussion of the study protocol where it is relevant for this analysis is provided here.

Measurements were obtained from the right femoral artery, right carotid artery and the right internal jugular vein for monitoring the intravascular pressures and cardiac output. Pulmonary artery pressure was recorded using a PAC (Swan-Ganz, CCO mbo $\mathrm{CCO} / \mathrm{SvO}_{2} 7.5 \mathrm{~F}$, Edwards Lifesciences, Irvine, CA) and esophageal pressure was measured via a latex ballon catheter (Viasys Healthcare, Hochberg, Germany). The esophageal catheters were connected to transducers (T450545A, Edwards Lifesciences, Irvine, CA) and the signals were transferred to a monitor (CM4008, Cardiomed, Ontario, 
Canada). Data were continuously measured and stored on a computer using Matlab (The MathWorks, MA). Analysis was performed using Matlab. Table 1 gives an overview of the measurements and signals used for the pig-specific parameter identification.

\subsubsection{Interventions}

The measurements were performed at three different PEEP levels of 0,10 and $20 \mathrm{~cm} \mathrm{H}_{2} \mathrm{O}$ and at 5 different volemic levels: baseline (normovolemia, N), $10 \%$ volume loss (hypovolemia, H), re-transfusion of blood (normovolemia, I1), infusion of a volume equal to $10 \%$ of the estimated blood volume (10\% hypervolemia, I2) and infusion of a volume equal to $20 \%$ of the estimated blood volume (20\% hypervolemia, I2). Each blood infusion or removal was

done over a period of 5 minutes, followed by a 10 minute stabilization period with $5 \mathrm{~cm} \mathrm{H}_{2} \mathrm{O}$ PEEP to guarantee steady-state measurements.

\section{Results}

\subsection{PEEP titration experiment at different volemic levels}

The integral-based parameter identification is applied to clinical porcine data for clinical model validation. Detailed results for three of the seven pigs are 
presented and a summary of all seven pigs is given in Table 2. Figure 4 shows in the upper panel of the Pressures panel the simulated model output for the systolic and diastolic pressures in the aorta (SAP and DAP, solid line). The circles are the measured systolic and diastolic pressures. Note, that the $\mathrm{x}$-axis shows the interventions performed during the PEEP titration protocol, where the $0-20$ range represent the beginning of a PEEP sequence of 0,10 and $20 \mathrm{~cm} \mathrm{H}_{2} \mathrm{O}$. The symbols $\mathrm{N}, \mathrm{H}, \mathrm{I} 1, \mathrm{I} 2$ and I3 stand for baseline normovolemia $(\mathrm{N})$, hypovolemia $(\mathrm{H})$, re-transfusion normovolemia (I1), 10\%and $20 \%$ hypervolemia (I2 and I3), respectively. The middle panel shows the systolic and diastolic pulmonary artery pressures (SPAP and DPAP) and the lower panel shows the central venous pressure (CVP). The simulation data match the measured porcine data well with absolute errors well below $5 \%$ for the maximum and minimum pressures.

Figure 4 displays in the upper panel of the Volumes panel the measured GEDV (one measurment/circle per intervention) and the simulated ventricular end-diastolic volume VEDV (VEDV=LVEDV+RVEDV, upper solid line). Note that the plotted GEDV is the corrected GEDV that has been multiplied with a correction factor of $2 / 3$. In addition, as the atria volumes are not included in this model, VEDV is multiplied by a factor of 1.6 (160\% of 
VEDV equals GEDV) to enable a direct comparison between the two volumes. The second line in the upper panel represents measured PBV (one measurment/circle per intervention) and the solid line represents the simulated pulmonary venous and capillary blood volume. Finally, the lower panel shows the measured (circles) and simulated SV (solid line). In all cases, the simulation data match the measured porcine data well with absolute errors well below $10 \%$ for the maximum and minimum volumes.

Figure 5 shows the simulated vs clinally measured pressure and volume signals for pig \#3 and Figure 6 shows the same results for pig \#5. Similar trends and good results are seen for all other pigs, as can also be seen in Table 2 .

Figure 7 clearly shows that the identified subject (pig) specific parameters for the venous return resistance $\left(R_{v r}\right)$ and pulmonary vascular resistance $\left(R_{\text {pulin }}\right)$, differ significantly between the $\mathrm{PEEP}=0 \mathrm{~cm} \mathrm{H}_{2} \mathrm{O}$ and $\mathrm{PEEP}=20 \mathrm{~cm} \mathrm{H}_{2} \mathrm{O}$ states. For all seven pigs, $R_{v r}$ increases when PEEP is applied. This increase is particularly significant during the hypo- and normo-volemia (H, N and I1) cases. Less significant changes are observed during hypervolemia (I2 and I3). Likewise, $R_{\text {pulin }}$ increases with increasing levels of PEEP with similar trends.

Figure 8 shows how $R_{v r}$ directly correlates with $P_{s y s} / S V$ for all 96 iden- 
tified segments and similar good correlations are obtained for the systemic vascular resistance $\left(R_{\text {sys }}\right)$ and $\left(S A P-P_{\text {sys }}\right) / S V$, and for $R_{\text {pulin }}$ and $(S P A P-$ $\left.P_{c a p}\right) / S V$, respectively. In all three cases, the correlations are tight $(\mathrm{r}=0.8-$ 0.99) with few outliers, indicating clear correlation, as expected.

Finally, Figure 9 shows the correlations for the ventricle contractilities $E_{\text {eslvf }}, E_{\text {esrvf }}$ and the ratio of systolic arterial pressure and end-systolic volume. Very tight correlations are obtained in both of these cases, as well.

\section{Discussion}

Mechanical ventilation, for example PPV with additional PEEP, can cause profound steady-state hemodynamic effects in critically ill patients. As respiratoryinduced changes in $P_{t h}$ are transmitted directly to all vessels in the thoracic cavity, the pressure in the right atrium increases during PPV. It is also known that PEEP often reduces venous return (VR) and thus cardiac output (CO) $[5,6,21]$

Over the years there has been some controversy how exactly PEEP alters VR. Some investigators have concluded that PEEP decreases VR by reducing the pressure gradient $\left(P_{s y s}-P_{r a}\right)[22]$. Note, that $P_{\text {sys }}$ is the mean systemic pressure, which is also known as mean circulatory filling pressure (MCFP). 
Since this CVS model does not explicitly model the right atrium, but lumps it together with the vena cava, $P_{r a}$ is represented in this model by $P_{v c}$. However, other researchers have demonstrated that $P_{\text {sys }}$ and $P_{r a}$ change equally during positive airway pressure and thus a change in the driving pressure gradient cannot be the reason for decreases in VR during PEEP [5, 23, 24]. Recent publications suggest that the main effect by which PEEP decreases venous return is by increasing the resistance to venous return $[5,6,21]$.

Thus, during PEEP, VR is decreased not by altering the pressure gradient $\left(P_{s y s}-P_{r a}\right)$, but by increasing the resistance to venous return, modelled here as $R_{v r}$. Hence, different PEEP levels should result in different values for $R_{v r}$. More specifically, $R_{v r}$ should increase during elevated PEEP levels, which is shown in Figure 7 and matches physiological expectations [5, 23, 24]. Furthermore, it is also known that right ventricular afterload increases during PPV [6], so one would expect $R_{\text {pulin }}$ to also increase with increasing levels of PEEP. Figure 7 shows this result, as expected.

Additionally, reflex response mechanisms of the body act to decrease unstressed systemic volume $\left(V_{d s y s}\right)$ and thus increase the stressed systemic volume $\left(V_{\text {sys }}\right)$, which in turn leads to a rise in $P_{\text {sys }}$ and helps to sustain the pressure gradient for VR $[25,26]$. The CVS model and identification method 
successfully capture this behavior, as can be seen in Figure 10. Figure 10 displays, how the effective systemic volume $V_{\text {sys }} f f\left(\right.$ with $\left.V_{\text {sys }} f f=V_{\text {sys }}-V_{\text {dsys }}\right)$ rises with PEEP levels from 0 to $20 \mathrm{~cm} \mathrm{H}_{2} \mathrm{O}$. Figure 10 shows the systemic volume $V_{\text {sys }}$ normalized by the baseline value for PEEP $0 \mathrm{~cm} \mathrm{H}_{2} \mathrm{O}$. The increase in $V_{\text {sys }}$ is more accentuated during hypo-(H) and normo(N,I1)-volemia, which is expected because the central venous pressure and intrathoracic pressure are both higher during these states compared to the 10\%- and $20 \%$ hypervolemia (I2,I3). Note, that PBV also decreases with rising PEEP levels, as the volume is shifted from the pulmonary circulation centrally to help raise $V_{\text {sys }}[25]$ as seen in the volume panels of Figures $4-6$.

In the pressure panels of Figures 4 - 6 it can be seen how well the increase in CVP is matched by simulated data from the CVS model for the different PEEP levels. Furthermore, Figure 8 shows that $R_{v r}$ correlates directly with the ratio of $P_{\text {sys }}$ and stroke volume, which is expected as the pressure gradient $\left(P_{s y s}-P_{v c}\right)$ divided by SV approximates the mean resistance to venous return. Note, that as the pressure gradient remains relatively equal and both pressures change similarly, it does not matter if $\left(P_{s y s}-P_{v c}\right) / S V$ or $P_{s y s} / S V$ is calculated.

Similar good correlations for $R_{\text {sys }}$ and $R_{\text {pulin }}$ are obtained as shown in 
Figure 8. These correlations further validate the CVS model and identification method as the identified parameters behave as expected based on physiological observations. Similarly, good correlations for the two ventricle contractilities $\left(E_{\text {eslvf }}, E_{\text {esrvf }}\right)$ are given in Figure 9, and are also expected physiologically. In this model, $V_{d}$ (ESPVR and x-axis intercept) is set to zero for simplicity. Thus, one can see that ESPVR is approximated by the ratio of systolic arterial pressure to end-systolic volume (SAP/ESV).

Finally, Table 2 summarizes all the results for all seven pigs over a total of 96 separate identification cases. Note, that 9 measurements had to be omitted from the final analysis as they contained corrupted data, such as those produced by disconnected catheters. These summary results show that the minimal CVS model is able to capture the essential dynamics of the porcine CVS response to increasing levels of PEEP over a variety of volemic conditions and over a selection of subjects. The minimal errors and strong correlations seen in this table and other figures indicate that the parameters are also behaving physiologically and not merely matching the data. 


\section{Conclusion}

The extended CVS model has successfully been shown to accurately simulate the impact different PEEP and volemic levels have on the hemodynamic status of the pigs. More specifically, separating the arterial and venous sides of both, systemic and pulmonary, circulation improved the model accuracy in simulating mechanical ventilation, where a reduction in LV afterload can be present along with a reduction in RV preload, thus requiring a reduction in $R_{\text {sys }}$, however a simultaneous increase in $R_{v r}$. This would not have been possible in the previous CVS model.

The integral-based optimization successively identified pig-specific parameters for the minimal cardiac model for a series of PEEP and volemic interventions. This validation study shows the ability of the model to adequately and realistically capture (with unique values) the impact of pressurevolume changes with PEEP and fluid therapy. The study cohort of seven porcine experiments may not be sufficient to truly validate the model and methods and further experiments are planned. However, while a full clinical validation of the model and further evaluation of the system are required, the model shows great potential and increases the confidence in the clinical applicability and validity of the developed methods and their use in clinical 
diagnostic monitoring.

\section{References}

[1] B. W. Smith, S. Andreassen, G. M. Shaw, P. L. Jensen, S. E. Rees, and J. G. Chase. Simulation of cardiovascular system diseases by including the autonomic nervous system into a minimal model. Comput Methods Programs Biomed, 86(2):153-160, May 2007.

[2] C. Starfinger, C. E. Hann, J. G. Chase, T. Desaive, A. Ghuysen, and G. M. Shaw. Model-based cardiac diagnosis of pulmonary embolism. Comput Methods Programs Biomed, 87(1):46-60, Jul 2007.

[3] B. W. Smith, J. G. Chase, R. I. Nokes, G. M. Shaw, and G. Wake. Minimal haemodynamic system model including ventricular interaction and valve dynamics. Medical Engineering 8 Physics, 26(2):131-139, 2004.

[4] C. E. Hann, J. G. Chase, and G. M. Shaw. Efficient implementation of non-linear valve law and ventricular interaction dynamics in the minimal cardiac model. Comput Methods Programs Biomed, 80(1):65-74, Oct 2005. 
[5] H.E. Fessler. Heart-lung interactions: applications in the critically ill. European Respiratory Journal, Series 'Clinical Physiology in Respiratory Intensive Care', 10(12):226-237, 1997.

[6] A.M. Miro and M.R. Pinsky. Pediatric Critical Care, chapter 24, pages 249-256. Mosby, 3 edition, 2005.

[7] C. E. Hann, J. G. Chase, and G. M. Shaw. Integral-based identification of patient specific parameters for a minimal cardiac model. Comput Methods Programs Biomed, 81(2):181-192, Feb 2006.

[8] D. Chemla, J.-L. Hébert, C. Coirault, K. Zamani, I. Suard, P. Colin, and Y. Lecarpentier. Total arterial compliance estimated by stroke volumeto-aortic pulse pressure ratio in humans. Am. J. Physiology (Heart and Circulation Physiology), 274:H500-H505, 1998.

[9] Y. Zhu, J. Dai, and J. K.-J. Li. Total systemic arterial compliance: evaluation of time and frequency domain methods. Bioengineering Conference, Proceedings of the 1995 IEEE 21 Annual Northeast, 1995.

[10] C. F. Rothe. Reflex control of the veins in cardiovascular function. Physiologist, 22(2):28-35, Apr 1979. 
[11] P. Neumann. Extravascular lung water and intrathoracic blood volume: double versus single indicator dilution technique. Intensive Care Med, 25(2):216-219, Feb 1999.

[12] S. Preisman, E. DiSegni, Z. Vered, and A. Perel. Left ventricular preload and function during graded haemorrhage and retranfusion in pigs: analysis of arterial pressure waveform and correlation with echocardiography. British Journal of Anaesthesia, 88(5):716-718, 2002.

[13] M. Nirmalan, M. Niranjan, T. Willard, J. D. Edwards, R. A. Little, and P. M. Dark. Estimation of errors in determining intrathoracic blood volume using thermal dilution in pigs with acute lung injury and haemorrhage. Br J Anaesth, 93(4):546-551, Oct 2004.

[14] Thomas Luecke, Harry Roth, Peter Herrmann, Alf Joachim, Gerald Weisser, Paolo Pelosi, and Michael Quintel. Assessment of cardiac preload and left ventricular function under increasing levels of positive end-expiratory pressure. Intensive Care Med, 30(1):119-126, Jan 2004.

[15] E. J. Kraut, J. T. Owings, J. T. Anderson, L. Hanowell, and P. Moore. Right ventricular volumes overestimate left ventricular preload in critically ill patients. J Trauma, 42(5):839-45; discussion 845-6, May 1997. 
[16] J. W. Kennedy, W. A. Baxley, M. M. Figley, H. T. Dodge, and J. R. Blackmon. Quantitative angiocardiography. i. the normal left ventricle in man. Circulation, 34(2):272-278, Aug 1966.

[17] T. Akiba, M. Nakasato, S. Sato, and A. Kikai. Angiographic determination of left and right ventricular volumes and left ventricular mass in normal infants and children. Tohoku J Exp Med, 177(2):153-160, Oct 1995.

[18] Daniel J. Schneck. The Biomedical Engineering Handbook, chapter 1 - An Outline of Cardiovascular Structure and Function, pages 1-9. Springer, 2000.

[19] Pulsion medical systems, munich germany: Picco plus user presentation, February 2005.

[20] P. Lambert, E. Sloth, B. Smith, L. K. Hansen, J. Koefoed-Nielsen, E. Tonnesen, and A. Larsson. Does a positive end-expiratory pressureinduced reduction in stroke volume indicate preload responsiveness? an experimental study. Acta Anaesthesiol Scand, 51(4):415-425, Apr 2007.

[21] L. Shekerdemian and D. Bohn. Cardiovascular effects of mechanical ventilation. Arch. Dis. Child, 80:475-480, 1990. 
[22] W.E. Johnston, J. Vinten-Johansen, W.P. Santamore, L.D. Case, and W.C. Little. Mechanism of reduced cardiac output during positive endexpiratory pressure in the dog. Am Rev Respir Dis, 140:1257-1264, 1989.

[23] H. Jellinek, H. Krenn, W. Oczenski, F. Veit, S. Schwarz, and R.D. Fitzgerald. Influence of positive airway pressure on the pressure gradient for venous return in humans. Journal of Applied Physiology, 88: 926-932, 2000.

[24] J.K. Peters, G. Lister, E.R. Nadel, and G.W. Mack. Venous and arterial reflex responses to positive-pressure breathing and lower body negative pressure. J Applied Physiology, 82(6):1889-1896, 1997.

[25] J. Peters, G.W. Mack, and G. Lister. The importance of the peripheral circulation in critical illness. Intensive Care Medicine, 27:1446-1458, 2001.

[26] E. Jacobsohn, R. Chorn, and M. O'Connor. The role of the vasculature in regulating venous return and cardiac output:: historical and graphical approach. Can J Anaesth, 44(8):849-867, 1977. 




Figure 1: Extended CVS model overview which includes additional compartments $P, V_{\text {sys }}$ and $P, V_{\text {cap }}$ to differentiate the arterial and venous sides of the pulmonary and systemic circulation. 

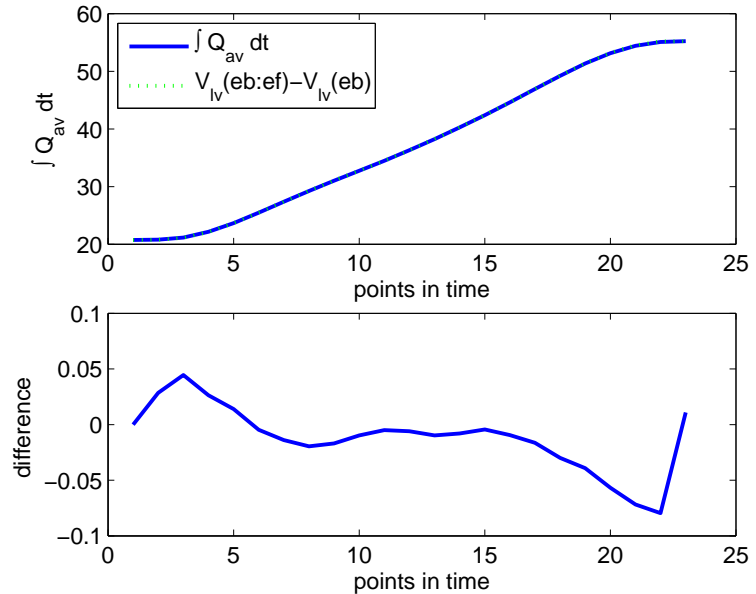

Figure 2: Substitution of flow signal with volume signal during identification process (upper panel) and difference between these two signals (lower panel). 


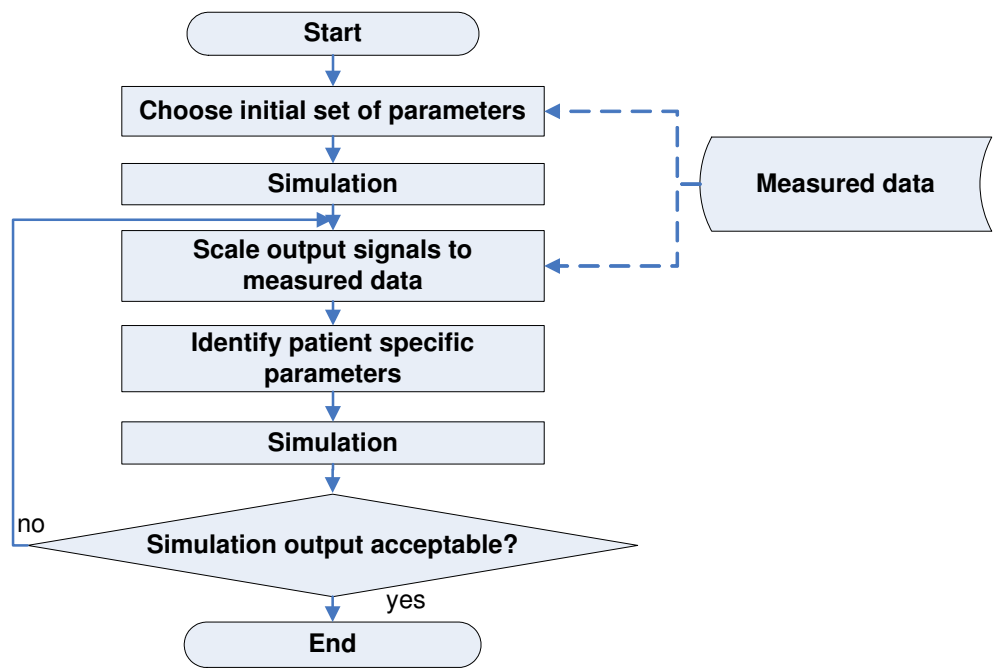

Figure 3: Parameter identification algorithm: 1.) a set of parameters is used for an initial simulation, 2.) data are then scaled to match the measured data and 3.) identified. This process is iterated until the simulation output is acceptable. 


\begin{tabular}{|l|l|l|}
\hline \multicolumn{2}{|l|}{ Measurements used for parameter ID } & Comments \\
\hline Signal & Obtained from & \\
\hline $\begin{array}{l}\text { stoke volume } \\
\text { SV }\end{array}$ & $\begin{array}{l}\text { PiCCO monitor } \\
\text { (Pusion Medical Systems, } \\
\text { Munich, Germany) }\end{array}$ & systolic and diastolic values \\
\hline $\begin{array}{l}\text { arterial pressure } \\
\text { (APsys, APdia) }\end{array}$ & PiCCO monitor \\
\hline $\begin{array}{l}\text { pulmonary artery pressure } \\
\text { (PAPmax, PAPmin) }\end{array}$ & $\begin{array}{l}\text { SC9000 monitor } \\
\text { (Siemens Medical Systems, } \\
\text { Germany) }\end{array}$ & systolic and diastolic values \\
\hline $\begin{array}{l}\text { central venous pressure } \\
\text { (CVPmean) }\end{array}$ & SC9000 monitor & mean value \\
\hline heart rate & PiCCO monitor & corrected by 2/3 \\
\hline $\begin{array}{l}\text { global end-diastolic volume } \\
\text { (GEDV) }\end{array}$ & PiCCO monitor & estimated as GEDV/4 \\
\hline $\begin{array}{l}\text { pulmonary blood volume } \\
\text { (PBV) }\end{array}$ & PiCCO monitor & mean value \\
\hline $\begin{array}{l}\text { esophageal pressure } \\
\text { (Pesomean) }\end{array}$ & $\begin{array}{l}\text { CM4008 monitor } \\
\text { (Cardiomed, Ontario, } \\
\text { Canada) }\end{array}$ & \\
\hline
\end{tabular}

Table 1: Signals that are used in the identification process to obtain the pig-specific parameters and to match the output signals of the CVS forward simulations to.

\begin{tabular}{|c|l|l|l|l|l|l|}
\hline \multicolumn{6}{|c|}{ Difference in \% for measured and simulated pressures and volumes } \\
\hline & SAP & DAP & SPAP & DPAP & CVP & SV \\
\hline$\mu$ & 3.57 & 5.51 & 3.79 & 4.98 & 3.55 & 5.35 \\
$\sigma$ & 2.22 & 3.34 & 2.36 & 3.35 & 2.36 & 3.38 \\
\hline
\end{tabular}

Table 2: Mean error and standard deviation in \% for measured and simulated pressures and volumes over all 96 identified segments. SAP = systolic arterial pressure, $\mathrm{DAP}=$ diastolic arterial pressure, $\mathrm{SPAP}=$ systolic pulmonary artery pressure, DPAP $=$ diastolic pulmonary artery pressure, $\mathrm{CVP}=$ central venous pressure, $\mathrm{SV}=$ stroke volume. 

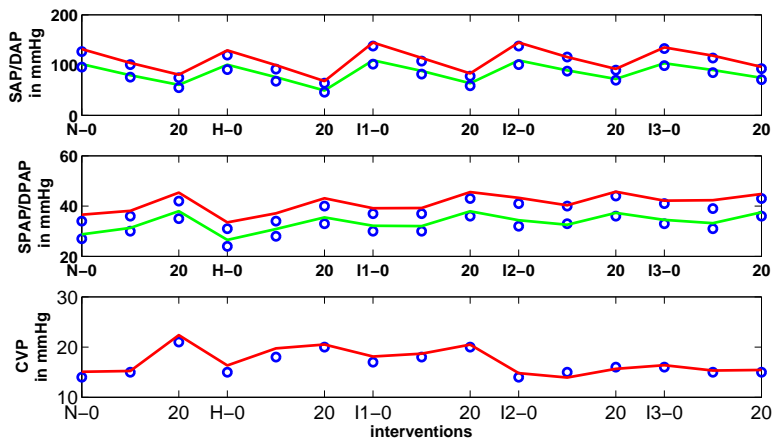

(a) Pressures
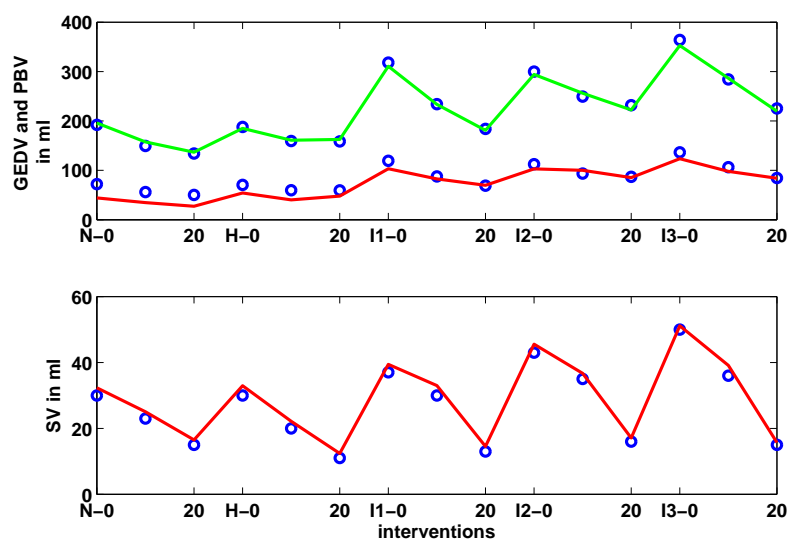

(b) Volumes

Figure 4: Pig 1, Model output (solid lines) vs clinical (circles) data for pressures and volumes. For the pressures, the upper panel shows systolic and diastolic arterial pressures (SAP, DAP), the middle panel shows systolic and diastolic pulmonary artery pressures (SPAP, DPAP) and the lower panel shows the central venous pressure (CVP). For the volumes, the upper panel shows the GEDV and PBV and the lower panel shows the stroke volume $(\mathrm{SV})$. 

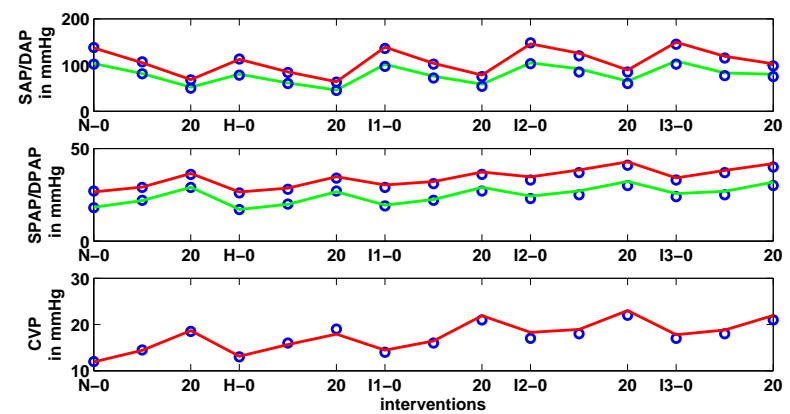

(a) Pressures
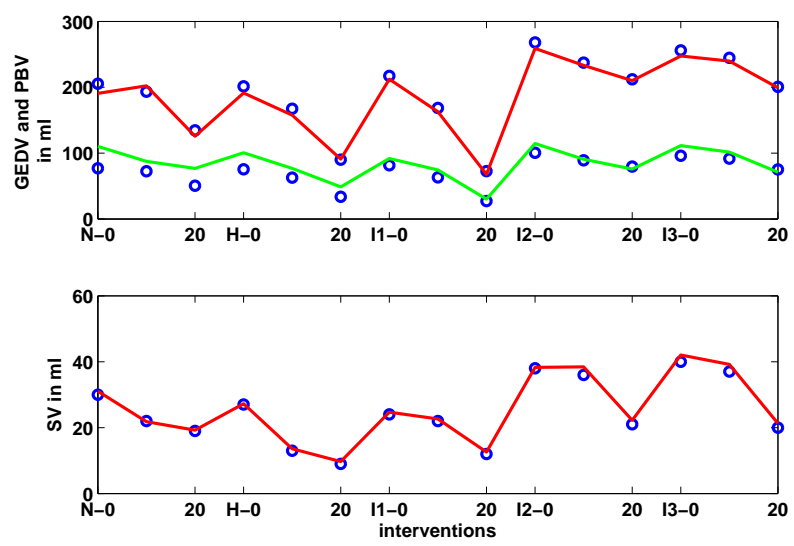

(b) Volumes

Figure 5: Pig 3, Model output (solid lines) vs clinical (circles) data for pressures and volumes. For the pressures, the upper panel shows systolic and diastolic arterial pressures (SAP, DAP), the middle panel shows systolic and diastolic pulmonary artery pressures (SPAP, DPAP) and the lower panel shows the central venous pressure (CVP). For the volumes, the upper panel shows the GEDV and PBV and the lower panel shows the stroke volume $(\mathrm{SV})$. 

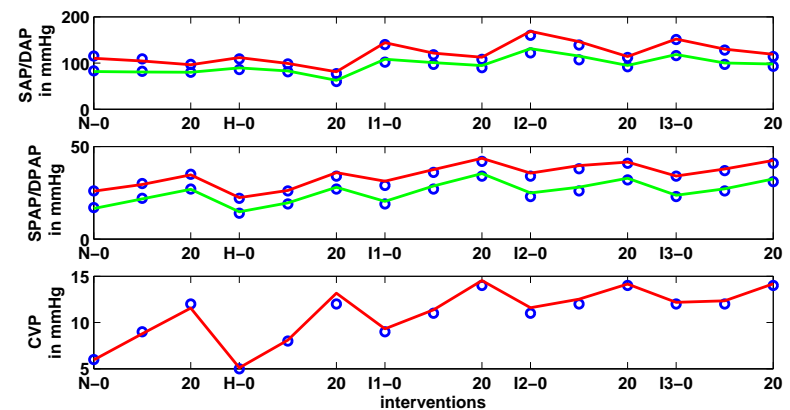

(a) Pressures
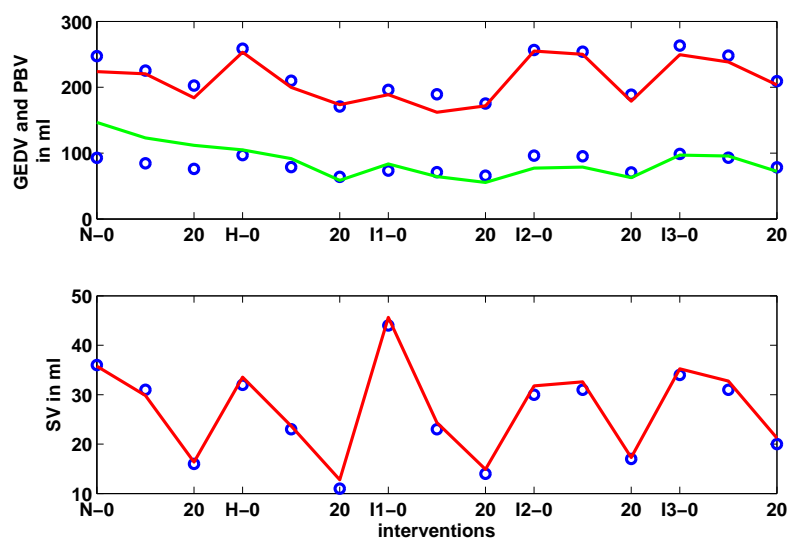

(b) Volumes

Figure 6: Pig 5, Model output (solid lines) vs clinical (circles) data for pressures and volumes. For the pressures, the upper panel shows systolic and diastolic arterial pressures (SAP, DAP), the middle panel shows systolic and diastolic pulmonary artery pressures (SPAP, DPAP) and the lower panel shows the central venous pressure (CVP). For the volumes, the upper panel shows the GEDV and PBV and the lower panel shows the stroke volume $(\mathrm{SV})$. 


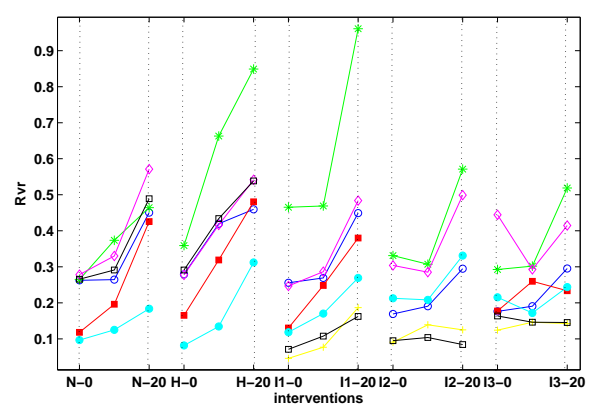

(a) $R_{v r}$

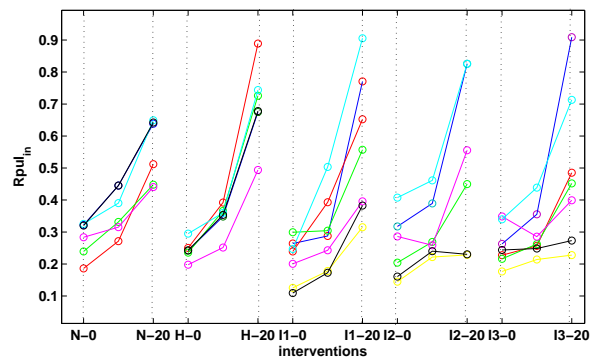

(b) $R_{\text {pulin }}$

Figure 7: Identified $R_{v r}$ and $R_{\text {pulin }}$ values for all 7 pigs and all PEEP/volume interventions. 


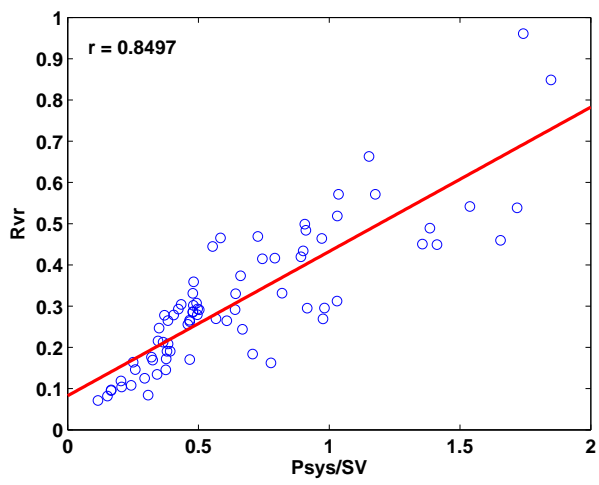

(a) $P_{s y s} / S V$ and $R_{v r}$

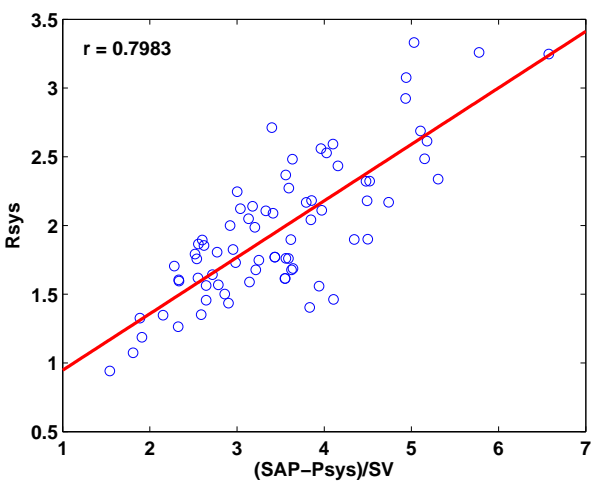

(b) $\left(S A P-P_{\text {sys }}\right) / S V$ and $R_{\text {sys }}$

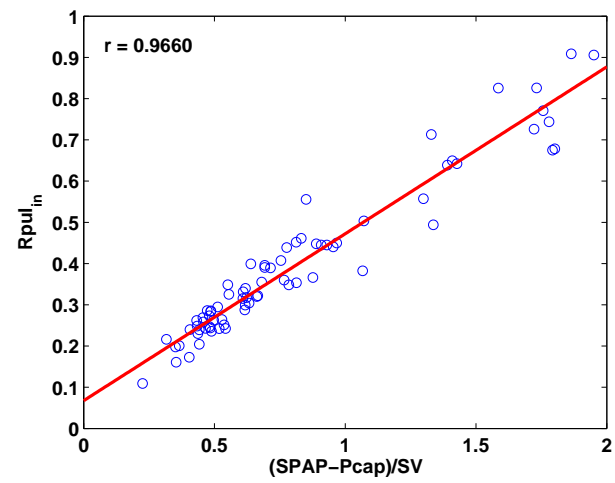

(c) $\left(S P A P-P_{\text {c(mp) }}\right) / S V$ and $R_{\text {pulin }}$

Figure 8: Correlation for all 96 identified segments for all 7 pigs and all $\mathrm{PEEP} /$ volume interventions. 


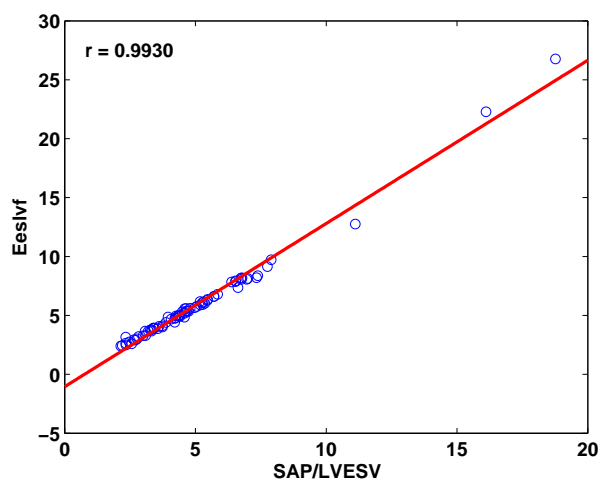

(a) $S A P / L V E S V$ and $E_{e s l v f}$

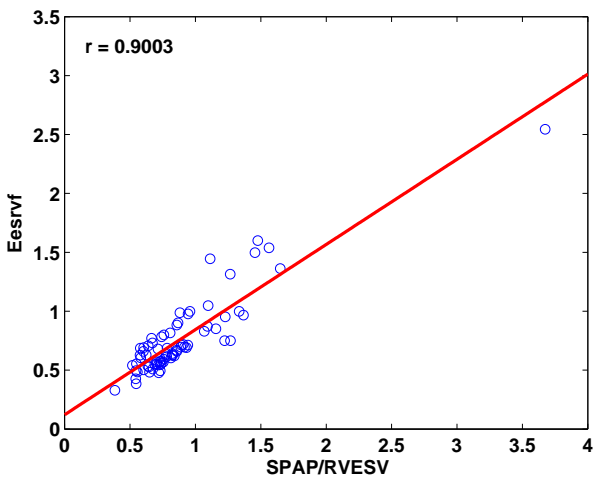

(b) $S P A P / R V E S V$ and $E_{\text {esrvf }}$

Figure 9: Correlation for $S A P / L V E S V$ vs $E_{\text {eslvf }}$ and $S P A P / R V E S V$ vs $E_{\text {esrvf }}$ for all 96 identified segments for all 7 pigs and all PEEP/volume interventions. 


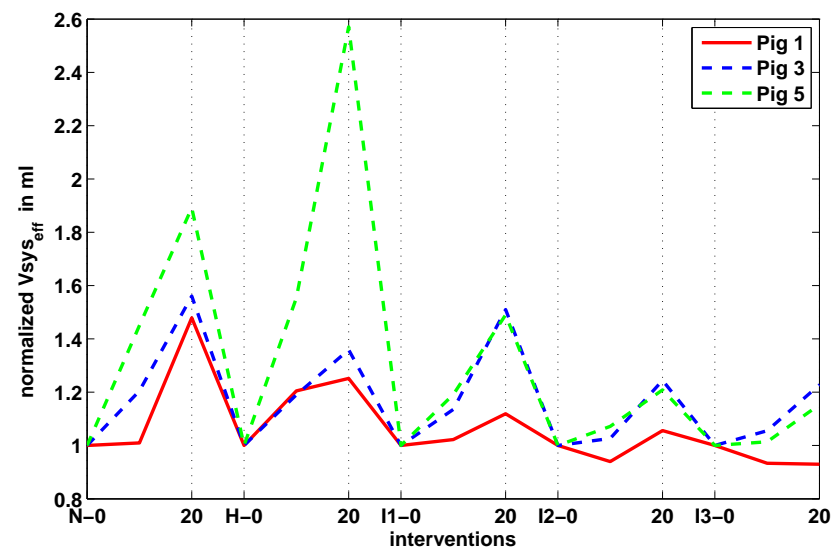

Figure 10: Effective normalized systemic volume for all 15 identified segments for pigs 1,3 and 5 over all PEEP/volume interventions. 